\title{
Direct Determination of Vibrational Density of States Change on Ligand Binding to a Protein
}

\author{
Erika Balog, ${ }^{1, *}$ Torsten Becker, ${ }^{1}$ Martin Oettl, ${ }^{2}$ Ruep Lechner, ${ }^{3}$ Roy Daniel, ${ }^{2}$ John Finney, ${ }^{4}$ and Jeremy C. Smith ${ }^{1, \dagger}$ \\ ${ }^{1}$ Computational Molecular Biophysics, IWR, Universität Heidelberg, Im Neuenheimer Feld 368, 69120 Heidelberg, Germany \\ ${ }^{2}$ Department of Biological Sciences, University of Waikato, Private Bag 3105 Hamilton, New Zealand \\ ${ }^{3}$ Hahn Meitner Institut Berlin, BENSC, Glienicker strasse 100, 14109 Berlin, Germany \\ ${ }^{4}$ Department of Physics and Astronomy, University College London, London WCIE 6BT, United Kingdom
}

(Received 18 September 2003; published 9 July 2004)

\begin{abstract}
The change in the vibrational density of states of a protein (dihydrofolate reductase) on binding a ligand (methotrexate) is determined using inelastic neutron scattering. The vibrations of the complex soften significantly relative to the unbound protein. The resulting free-energy change, which is directly determined by the density of states change, is found to contribute significantly to the binding equilibrium.
\end{abstract}

DOI: 10.1103/PhysRevLett.93.028103

PACS numbers: $87.15 . \mathrm{He}$

An understanding of how ligands bind to proteins is of fundamental importance in biology and medicine [1-6]. Protein:ligand association has been assumed to be dominated by factors such as the hydrophobic effect, hydrogen bonding, electrostatic, and van der Waals interactions. However, as early as 1963 it was suggested that an additional mechanism might exist, due to increased flexibility in the protein:ligand complex manifested by a change in the spectrum of vibrations due to formation in the complex of new, intermolecular interactions [7-13]. Theoretical normal mode analyses, used to estimate this vibrational change on insulin dimerization [12] and on water binding to bovine pancreatic trypsin inhibitor [13], have suggested that the effect is likely to be thermodynamically important. However, experimental determination of the vibrational change has been lacking.

Inelastic neutron scattering, in which the dynamic structure factor $S(q, \omega)$ is measured as a function of the scattering wave vector $q$ and energy transfer $\hbar \omega$ (where $\omega$ is the angular frequency), has been used to determine the vibrational density of states (frequency distribution) $g(\omega)$ for several proteins [14-16]. Here we present an experimental determination of the change in $g(\omega)$ on binding a ligand to a protein. This determination allows thermodynamic quantities associated with the vibrational change to be derived. The enzyme chosen is dihydrofolate reductase (DHFR), an important target for anticancer and antibacterial drugs [17-21]. DHFR catalyzes the reduction of dihydrofolate to tetrahydrofolate in the presence of the nicotinamide adenine dinucleotide phosphate (NADPH) cofactor. The ligand used is methotrexate (MTX), a folate antagonist of DHFR that has been used effectively as a cytotoxic agent in the treatment of cancers [22].

To minimize scattering from solvent molecules, the system was exchanged with $\mathrm{D}_{2} \mathrm{O}$. To do this, lyophilized DHFR from E.coli was dissolved in $\mathrm{D}_{2} \mathrm{O}$ equilibrated at $4{ }^{\circ} \mathrm{C}$ overnight and freeze dried. NADPH and NADPH +
MTX were added in equimolar ratios to the enzyme. As the dissociation constants of DHFR with NADPH and MTX are low $\left(K_{d}^{\mathrm{NADPH}}=0.01 \mu \mathrm{M}, K_{d}^{\mathrm{MTX}}=0.15 \mu \mathrm{M}\right.$ [23]), it can be assumed that the ligands bind quantitatively to the enzyme. $\mathrm{D}_{2} \mathrm{O}$ was added, the solutions equilibrated for $4 \mathrm{~h}$ at $4{ }^{\circ} \mathrm{C}$ and freeze dried, and a final exchange step involving dissolution in $\mathrm{D}_{2} \mathrm{O}$ and lyophilization was performed. The uncomplexed enzyme (DHFR + NADPH) and the complexed enzyme (DHFR + NADPH + MTX) were hydrated to a degree of $30 \%$, i.e., $30 \mathrm{mg}$ of $\mathrm{D}_{2} \mathrm{O}$ per $100 \mathrm{mg}$ of dry weight protein. This was performed by equilibrating the samples in a saturated solution atmosphere $\left(\mathrm{KBr}\right.$ in $\left.\mathrm{D}_{2} \mathrm{O}\right)$ at $20^{\circ} \mathrm{C}$ for 3 days.

For the neutron experiments, the samples were contained in an aluminum sample holder. Sample amounts were $98.1 \mathrm{mg}$ (uncomplexed) and $108.6 \mathrm{mg}$ (complexed). The measurements were performed on the time-of-flight spectrometer IN6 at the Institut Laue-Langevin (ILL), Grenoble, with an incident neutron beam wavelength of $5.12 \AA$. The scattering experiments were performed at $120 \mathrm{~K}$ to ensure that all dynamics present is harmonic. Accumulation times were $24 \mathrm{~h} /$ sample. Samples were oriented at $135^{\circ}$ with respect to the incident beam. Both sample transmissions were $97.7 \%$, high enough to ensure the absence of multiple scattering effects. The raw data were corrected using the INX program at ILL [24]. This program normalizes the detector responses with respect to an angle-independent standard vanadium sample, normalizes each data collection run to the number of incident neutrons, performs slab corrections for selfshielding, and subtracts the cell scattering from sample + cell scattering. To improve the statistics, the spectra derived from 122 scattering angles (from $10^{\circ}$ to $114^{\circ}$ ) were binned into 11 constant-angle spectra. In order to compare the relative differences in the inelastic part of the spectra of the two samples, dynamical structure factors were normalized to the elastic peak height. The errors in $S(q, \omega)$ (see Fig. 1) are statistical errors of the 


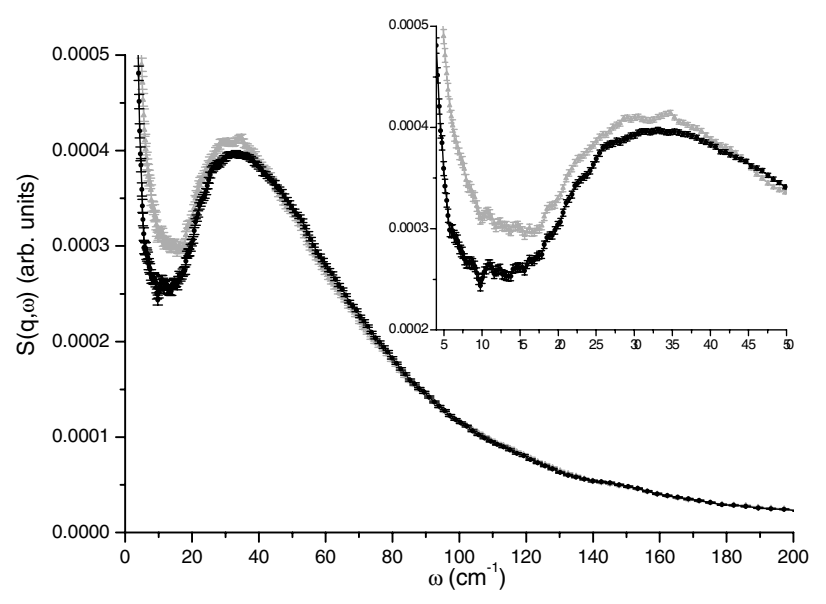

FIG. 1. Dynamic structure factor versus frequency for uncomplexed DHFR (black curve) and complexed with methotrexate form of DHFR (gray curve) at $120 \mathrm{~K}$. Data from all scattering angles are summed. Both spectra are normalized to the elastic peak height. Inset: the low frequency region of the spectra.

measurement. The errors in deduced quantities were determined by the error propagation.

The experimental vibrational frequency distribution $g_{\exp }(\omega)$ was obtained from the spectra using the limit $[14,16]$

$$
g_{\exp }(\omega)=\lim _{q \rightarrow 0} \frac{6 \omega}{\hbar q^{2}}\left(e^{\hbar \omega / k T}-1\right) S(q, \omega),
$$

where $\hbar$ is Planck's constant divided by $2 \pi, k$ is the Boltzmann constant, and $T$ is the temperature.

$g_{\text {exp }}(\omega)$ is on a relative scale. To determine the absolute density of states $g(\omega)$, use was made of the fact that, in the harmonic approximation at low $q$, the elastic scattering intensity $S(q, 0)$ is given by $S(q, 0)=\exp \left[-u^{2} q^{2} / 6\right]$, where $u^{2}$ is the mean-square displacement. Thus, $u^{2}$ can be determined from the gradient of $\ln S(q, 0)$ vs $q^{2}$. The corresponding mean-square displacement $u_{\text {calc }}^{2}$ calculated from $g_{\exp }(\omega)$ is obtained by integrating as follows: $u_{\text {calc }}^{2}=$ $\frac{\hbar}{2 M} \int g_{\exp }(\omega) \operatorname{coth}\left(\frac{\hbar \omega}{2 k T}\right) d \omega$, where $M$ is the protein mass. The absolute density of states can then be derived by normalizing as follows: $g(\omega)=\left(u^{2} / u_{\text {calc }}^{2}\right) g_{\exp }(\omega)$.

Figure 1 shows the dynamic structure factor $S(q, \omega)$ of the "uncomplexed" (DHFR + NADPH) and "complexed" (DHFR + NADPH + MTX) forms of the enzyme. A significant difference can be seen in the lowfrequency region of the spectra $\left(\omega\right.$ below $\sim 40 \mathrm{~cm}^{-1}$ ). The fact that $S(q, \omega)$ is higher for the complexed in the low-frequency region implies that the complexed form of the enzyme has a higher number of low-frequency modes [see Eq. (1)] and thus is more flexible than the uncomplexed form. This is confirmed by Fig. 2, which shows that, below $\sim 15-20 \mathrm{~cm}^{-1}, g(\omega)$ of the complexed form is significantly higher than that of the uncomplexed protein.

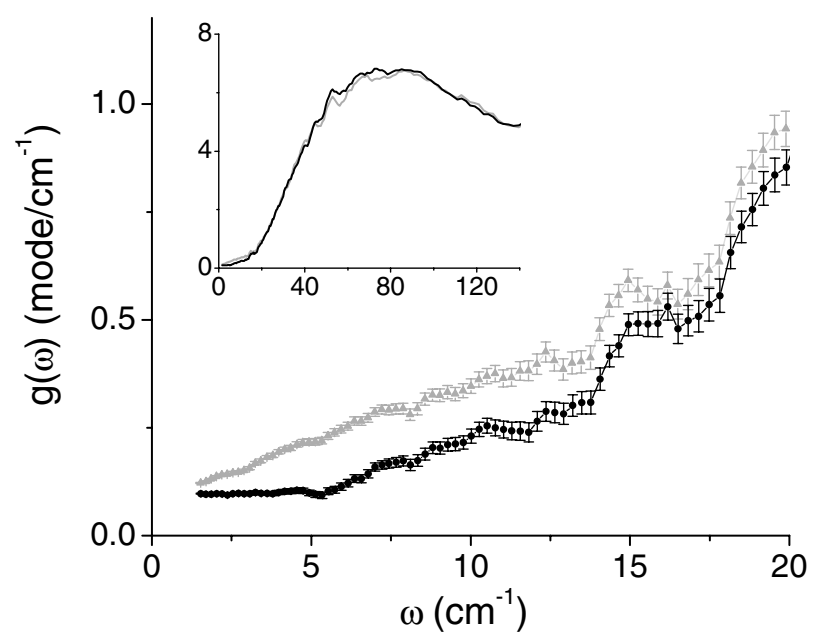

FIG. 2. Vibrational frequency distribution $g(\omega)$ for uncomplexed (black) and complexed (gray) forms of DHFR. Inset: enlarged frequency region of the spectra.

For the protein to soften on binding, there must be a shift in $g(\omega)$ from higher frequencies (in the uncomplexed protein) to lower frequencies (in the complexed). One possibility for this that would be consistent with Fig. 1 is that softened modes may be of too-high frequency in the uncomplexed protein to be detectable by the instrument. However, there is evidence of a frequency shift occurring within the frequency range shown in Fig. 2. Integration of the difference between $g(\omega)$ of the two samples shows that the net equivalent of about two modes are transferred from the $40-80 \mathrm{~cm}^{-1}$ region in the uncomplexed protein to the region below $20 \mathrm{~cm}^{-1}$ in the complexed.

The change in $g(\omega)$ over the range $40-80 \mathrm{~cm}^{-1}$ is not accompanied by a statistically significant change in the $S(q, \omega)$ in Fig. 1. The reason for this is that $S(q, \omega)$ is amplitude weighted. Thus, removal of a low-amplitude, higher-frequency mode from the spectrum leads to only a relatively small intensity loss, whereas adding a highamplitude, low-frequency mode adds a relatively large intensity. Consequently, the shifted modes scatter much more strongly in the complexed than in the uncomplexed molecule, with the net result that the spectral change appears as an increase in intensity at low frequencies in the complexed.

The vibrational partition function $Z_{\mathrm{vib}}$, i.e., the partition function associated with the vibrational energy levels in a harmonic system, is determined by $g(\omega)$ as follows [25]:

$$
Z_{\mathrm{vib}}=\prod_{i=1}^{3 N-6} \frac{e^{-\beta \hbar \omega_{i} / 2}}{1-e^{-\beta \hbar \omega_{i}}}=\prod_{\omega=\omega_{\min }}^{\infty}\left(\frac{e^{-\beta \hbar \omega / 2}}{1-e^{-\beta \hbar \omega}}\right)^{g(\omega)},
$$

where $\omega_{\min }$ is the lowest frequency in the system and $b=1 / k T$. The associated Helmholtz vibrational free energy $A_{\text {vib }}$ is given by $A_{\text {vib }}=-k T \ln Z_{\text {vib }}$, the energy by 
$\bar{E}_{\text {vib }}=-\frac{\partial}{\partial \beta} \ln \left(Z_{\text {vib }}\right)$ (this is the enthalpy change for a process for which the volume change can be neglected), and the entropy by $S_{\text {vib }}=k\left[\ln \left(Z_{\text {vib }}\right)+\beta \bar{E}\right]$.

The free-energy change $\Delta A_{\text {vib }}$ calculated from the density-of-states change in Fig. 2 is $-17 \pm 4 \mathrm{~kJ} / \mathrm{mol}$ at $300 \mathrm{~K}$, favoring binding. The free-energy change contains a large entropic contribution $-T \Delta S_{\text {vib }}(-25 \pm$ $6 \mathrm{~kJ} / \mathrm{mol}$ ) that is partially compensated by the enthalpy term $\Delta \bar{E}_{\mathrm{vib}}(8 \pm 2 \mathrm{~kJ} / \mathrm{mol})$ that opposes binding.

The thermodynamic results are subject to errors associated with the use of the harmonic approximation and the necessity of determining the dynamic structure factor on an absolute scale. The scattering measurements were performed at $120 \mathrm{~K}$. At this temperature the protein exhibits only harmonic vibrational dynamics [15], thus permitting the direct determination of the vibrational density of states.

The $300 \mathrm{~K}$ vibrational density of states of trehalosecoated myoglobin has been found to be identical to that at $100 \mathrm{~K}$, indicating harmonic behavior [26]. For hydrated proteins at physiological temperatures, a significant contribution to the atomic mean-square displacements arises from anharmonic dynamics [26-28]. These anharmonic motions, together with overdamping of the low-frequency modes, result in quasielastic scattering that renders difficult the derivation of model-independent thermodynamic quantities from experimental data [28]. It is conceivable that anharmonic degrees of freedom might also be modified on ligand binding. However, molecular dynamics simulations suggest that $\sim 99.5 \%$ of the modes in a protein are effectively harmonic at $300 \mathrm{~K}$ [29]. Assuming the protein does remain harmonic, the density-of-states change will be the same at 300 as it is at $120 \mathrm{~K}$.

The dynamical change seen here indicates softening of the protein on complexation. Normal mode calculations on proteins have indicated that the vibrational modes in the frequency range at which the change is seen here involve mainly collective displacements of groups of atoms distributed throughout the protein [30]. These modes may be modified by environmental effects, although in the present case the sample preparation is consistent with the environments of the complexed and the uncomplexed forms being the same. Normal mode calculations concur with the present findings in that they also indicate an increase in flexibility on ligand binding to proteins and protein:protein association [12,13]. Furthermore, increased backbone conformational flexibility on binding a hydrophobic ligand to mouse major urinary protein has been detected using nuclear magnetic resonance (NMR) relaxation experiments [31]. However, these results contrast with other observations using NMR and crystallography of a flexibility decrease on protein binding to small organic ligands [32-34]. NMR and crystallographic measurements are likely to be dominated by changes in anharmonic degrees of freedom not present in the conditions studied here. Moreover, the vibrational changes seen in the present work are not detectable using NMR or crystallography $[12,13]$.

There remains much to learn about the various contributions to ligand binding free energies. For example, although hydrogen bonds normally must be satisfied, whether they bring any net contribution to the binding free energy in aqueous solution is debatable. For the present system, although significant ligand:protein hydrogen bonding exists, free-energy perturbation calculations suggest that hydrophobic interactions contribute as much to the binding free energy as do hydrophilic [35]. Another effect that remains to be accurately quantified is the loss on binding of the whole-molecule translational and rotational entropic free energies of the protein and ligand [27,36-39].

The contribution elucidated here, that from vibrational changes, has been hitherto largely neglected in the consideration of binding equilibria, due largely to the absence of an experimental technique for determining it. The estimation of $-17 \pm 4 \mathrm{~kJ} / \mathrm{mol}$ for the vibrational free-energy change can be compared with the experimentally estimated total binding free energy in the present system, which is $39 \mathrm{~kJ} / \mathrm{mol}$ [23]. This suggests that the vibrational change is thermodynamically significant and would contribute a factor of $\sim 10^{3}$ to the binding constant. This illustrates how modifications of equilibrium internal fluctuations in a protein can have consequences for binding equilibria of biomedical importance. Recently, the vibrational effect has been included together with electrostatic, van der Waals, and nonpolar terms in an empirical theoretical analysis of ligand binding free energies [40]. Whether the vibrational softening effect seen here is general for protein:ligand interactions remains to be determined. The direct access to the vibrational density of states provided by inelastic neutron scattering holds promise for the study of vibrational thermodynamic changes in many biomolecular association processes.

We gratefully acknowledge Jacques Ollivier from the Institut Laue-Langevin for help with neutron experiments and Stefan Fischer for help and useful discussions.

*Present address: Institut de Hautes Etudes Scientifiques, 35 route de Chartres, 91440 Bures sur Yvette, France.

${ }^{\dagger}$ Corresponding author.

Electronic address: biocomputing@iwr.uni-heidelberg.de

[1] W. Wang et al., Annu. Rev. Biophys. Biomol. Struct. 30, 211 (2001).

[2] M. L. Lamb and W. L. Jorgensen, Curr. Opin. Chem. Biol. 1, 449 (1997).

[3] L. Y. Lian et al., Methods Enzymol. 239, 657 (1994).

[4] I. M. Klotz, Q. Rev. Biophys. 18, 227 (1985).

[5] S. J. Benkovic, C. A. Fierke, and A. M. Naylor, Science 239, 1105 (1988).

[6] M. K. Gilson et al., Biophys. J. 72, 1047 (1997). 
[7] I. Z. Steinberg and H. A. Scheraga, J. Biolumin. Chemilumin. 238, 172 (1963).

[8] M. I. Page and W. P. Jencks, Proc. Natl. Acad. Sci. U.S.A. 68, 1678 (1971).

[9] J. M. Sturtevant, Proc. Natl. Acad. Sci. U.S.A. 74, 2236 (1977).

[10] H. P. Erickson, J. Mol. Biol. 206, 465 (1989).

[11] A. V. Finkelstein and J. Janin, Protein Eng. 3, 1 (1989).

[12] B. Tidor and M. Karplus, J. Mol. Biol. 238, 405 (1994).

[13] S. Fischer, J. C. Smith, and C. S. Verma, J. Phys. Chem. B 105, 8050 (2001).

[14] S. Cusack et al., J. Mol. Biol. 202, 903 (1988).

[15] W. Doster, S. Cusack, and W. Petry, Nature (London) 337, 754 (1989).

[16] J. C. Smith et al., J. Chem. Phys. 85, 3636 (1986).

[17] M. R. Sawaya and J. Kraut, Biochemistry 36, 586 (1997).

[18] D. M. Epstein, S. J. Benkovic, and P.E. Wright, Biochemistry 34, 11037 (1995).

[19] E. E. Howell et al., Science 231, 1123 (1986).

[20] S. R. Stone and J. F. Morrison, Biochemistry 23, 2753 (1984).

[21] T. Kamiyama and K. Gekko, Biochim. Biophys. Acta 1478, 257 (2000).

[22] F. M. Huennekens, Adv. Enzyme Regul. 34, 397 (1994).

[23] J. Basran et al., Biochemistry 34, 2872 (1995).

[24] F. Rieutord, Institut Laue-Langevin Report No. 90RI17T, 1990.

[25] D. A. McQuarrie, Statistical Mechanics (Harper \& Row,
New York, 1976).

[26] L. Cordone et al., Biophys. J. 76, 1043 (1999).

[27] A. Tournier and J. C. Smith, Phys. Rev. Lett. 91, 208106 (2003).

[28] J. C. Smith et al., J. Chem. Phys. 93, 2974 (1990).

[29] A. Kitao, S. Hayward, and N. Go, Proteins 33, 496 (1998).

[30] B. Brooks and M. Karplus, Proc. Natl. Acad. Sci. U.S.A. 80, 6571 (1983).

[31] L. Zidek, M.V. Novotny, and M. J. Stone, Nat. Struct. Biol. 6, 1118 (1999).

[32] J.W. Cheng, C. A. Lepre, and J. M. Moore, Biochemistry 33, 4093 (1994).

[33] C. Rischel et al., Biochemistry 33, 13997 (1994).

[34] D. Fushman, O. Ohlenschlager, and H. Ruterjans, J. Biomol. Struct. Dyn. 11, 1377 (1994).

[35] U. Singh and S. Benkovic, Proc. Natl. Acad. Sci. U.S.A. 85, 9519 (1988).

[36] M. H. Zaman, R. S. Berry, and T. R. Sosnick, Proteins 48, 341 (1999).

[37] A. Tamura and P. L. Privalov, J. Mol. Biol. 273, 1048 (1997).

[38] M. Karplus and J. Janin, Protein Eng. 12, 185 (1999).

[39] Y. B. Yu, P. L. Privalov, and R. S. Hodges, Biophys. J. 81, 1632 (2001).

[40] S. Schwarzl, T. B. Tschopp, J. C. Smith, and S. Fischer, J. Comput. Chem. 23, 1143 (2002). 